\title{
The Clinical Performance and Effectiveness of the Multiple-Attending-Physicians System Compared with the Single-Attending-Physician System in Inpatient Setting: A Mixed-Method Study
}

\section{Daeho Park}

Daisen Clinic

Toshihiro Hamada ( $\nabla$ thamada@tottori-u.ac.jp)

Tottori University

Shintaro Imaoka

Tottori University

Shohei Taniguchi

Tottori Prefectural Central Hospital

Yuma Ohtsuka

Tottori Prefectural Central Hospital

Yu Wakunami

Tottori Prefectural Central Hospital

Young Lee

Tottori University

Minako Kamimoto

Tottori University

Kazuoki Inoue

Daisen Clinic

Daisuke Son

Tottori University

Hiromi Matsumoto

Kawasaki University of Medical Welfare

Takuya Shimosaka

Hino Hospital

Shuichi Sasaki

Hino Hospital

Youngee Lee

Hino Hospital

Masahiko Koda

Hino Hospital 


\section{Shin-ichi Taniguchi}

Tottori University

\section{Research Article}

Keywords: multiple-attending-physicians system, group practice, single-attending-physician system, inpatient care, primary care

Posted Date: July 27th, 2021

DOl: https://doi.org/10.21203/rs.3.rs-677680/v1

License: (c) (1) This work is licensed under a Creative Commons Attribution 4.0 International License. Read Full License 


\section{Abstract}

\section{Background}

Considering work styles especially of solo attending physicians, medical facilities have been required to effectively utilize sufficient human resources in many countries. Therefore, we compared crosssectionally clinical performance of the single- and the multiple-attending physicians groups in inpatient care. Furthermore, we also qualitatively analyzed physicians' working burden and quantitively assessed advantages / disadvantages of those attending physicians systems.

\section{Method}

In this cross-sectional study, we extract electronic health record of patients from a hospital in Japan from April 2017 to October 2018 to compare anonymous statistical data including average lengths of hospital stay and patient outcome between the single-attending and multiple-attending-physicians system. Then, we conducted a questionnaire survey for all physicians of single and multiple-attending physician systems, asking their physical and psychiatric working burden, and their reasons and comments on their working styles.

\section{Result}

The average length of hospital stay was significantly shorter in the multiple-attending physicians system than in the single-attending physicians system, while patients' age, gender and diagnoses were similar. From the questionnaire survey, although physical burden in multiple-attending physicians system tended to be lower than that in single-attending physician system, no significant difference was found in all categories. Advantages of multiple-attending physicians system extracted from qualitative analysis are 1) Improvement of physicians ' quality of life (QOL), 2) Lifelong-learning effect, and 3) Improving the quality of medical care, while disadvantages were 1) Risk of miscommunication, 2) Conflicting treatment policies among physicians, and 3) unfamiliar culture with multiple-attending physicians system.

\section{Conclusion}

Multiple-attending-physicians system in inpatient care may reduce physical burden on physicians, and not only improve QOL of physicians but lead to lifelong learning and improving the quality of medical care.

\section{Introduction}

Attending physicians have primary responsibility for the overall course of a patient's treatment in the ward or outpatient services and are expected to have the best understanding of the patient's physical health and other conditions. Under the multiple-attending-physicians system, one patient is assigned to a team of physicians who are authorized to make patient-care decisions, which means that the time and responsibility for the patient's medical treatment can be distributed among multiple physicians. In 
contrast, inpatient medical care in Japan has historically been provided under a single-attendingphysician system wherein one representative physician works with patients as the sole attending physician. Under this system, a single physician often bears responsibility for the management of a patient's condition even if another on-duty physician was working within the medical facility during holidays or after hours.

Since the physician population is gradually aging, especially in rural areas [1], medical institutions need to secure adequate human resources. Although the employment of primary care physicians has been identified as a potential solution to this issue, primary care physicians in rural areas have a high burnout rate, and proactive measures to mitigate this risk are important $[2,3,4]$. Recently, there has been a demand for reform in doctors' working style across Japan. The Panel on the Reform of Doctors' Work Style under the Ministry of Health, Labour, and Welfare proposed some measures to reduce the length of hours that doctors can work at medical facilities. For example, 1) not being obligated to explain the course of the disease for minor conditions (such as common cold) or provide advice to non-emergency patients on holidays or during after-hours, 2) reducing their post-night-shift duties, 3 ) having mandatory holidays, and 4) introducing a multiple-attending-physicians system $[5,6]$.

Therefore, the shared responsibility for inpatients may decrease emergency calls and reduce physical and psychiatric burdens on a single physician. The advantages and disadvantages of these two attendingphysician systems have not been investigated sufficiently [6]. According to a cross-sectional questionnaire survey in Japan, departments with multiple attending physicians had shorter overtime hours than those with single attending physicians, and there was no difference in job satisfaction [7]. In contrast, studies from German general practitioners in solo and group practices revealed a higher prevalence of emotional exertion, depersonalization, and little self-accomplishment among physicians in group practices compared to solo practices [8].

Therefore, the present study aimed to compare the clinical performance of physicians in inpatient care between the single-attending-physician system and the multiple-attending-physicians system, crosssectionally. Furthermore, we conducted a questionnaire survey on physicians who have worked under a single or multiple-attending-physicians system to qualitatively analyze their work burden and identify the advantages and disadvantages of the multiple-attending-physicians system.

\section{Materials And Methods}

The present study was performed at the Hino Hospital, which was the only hospital in the town of Hino, southwestern area of Tottori prefecture, Japan. The population of Hino Town was 3,278, and the elderly rate (individuals aged above 65 years) was 49.0\% in 2019. As of 2017, the Hino Hospital had 99 beds in two wards and nine full-time doctors (4 for Internal Medicine, 2 for Surgery, 1 each for Orthopedics, Ophthalmology, and Pediatrics) operating under the single-attending-physician system. Meanwhile, in June 2014, the Tottori University Center for Integrated Education and Training in Community Medicine was established in the Hino Hospital, and since then, one general physician as a part-time employee is 
sent from the Tottori University to the outpatient clinics in the hospital each day. In 2017, the number of full-time internal medicine physicians at the Hino Hospital was reduced from four to three, which led to the addition of one more general physician from the Tottori University to manage inpatient care in the Department of General Medicine. Since there were one to three part-time physicians on duty during weekdays, a different physician was in charge of the inpatient treatment for each day, rotating daily. After hours and on weekends, one physician from the attending physician team responded to the inpatients regarding changes in their condition. Thus, under the multiple-attending-physicians system, the general physicians in the Department of General Medicine treated inpatients within the wards where internal medicine physicians engaged in their practice.

In this study, we defined single-attending physician as solo attending physician who is in charge of, decide and treat all clinical issue by oneself, including off-duty hours and weekends, whereas multipleattending physician as two or more physicians share their responsibilities and clinical workloads for patients.

In this cross-sectional study, to compare the clinical performance between the groups of physicians under the single and multiple-attending-physician systems, we obtained anonymous statistical data from electronic medical records from April 2017 to October 2018. We collected information regarding patients' age, sex, length of hospital stay, living area, status before admission, main disease, prognosis, and total inpatient care costs. Simultaneously, the number of working physicians per unit time was aggregated to calculate the actual number of attending physicians for each group. We calculated the number of inpatients per actual working physicians from the number of actual working physicians in each group and the number of patients.

Next, we performed a qualitative study to determine the physicians' clinical competence and burden of work under the two systems and also compared the advantages and disadvantages of both systems. We surveyed 11 physicians, including 6 general physicians and 5 internal medicine physicians. At the start of this study, written informed consent was obtained from all physicians who were surveyed, and allowed that they could withdraw their consent or ask questions at any time. Two group of physicians were completely separated and not duplicated. The questionnaire contained 18 items, including physicians' identification (age, post-graduate year, sex, and the former experience of the multiple-attendingphysicians system). It also included the following items about their current working conditions that were assessed using a visual analog scale (VAS) [9]:

How many hours per week are spent on after-hours inpatient services? (After-hours will be considered before 8:30 a.m. and after 5 p.m.; after-hour working time per week); what is the physical and psychiatric burden of providing inpatient care? (Physical and psychiatric work burden); how well do you understand your patients' disease status? (Understanding of patients' disease status); how much of a trusting relationship do you have with your patients? (Doctor-patient relationship); how much imbalance do you feel in your work, such as focusing on yourself or other doctors? (Imbalances due to workload); how well do you collaborate with other professionals? (Inter-professional relationship). 
They also provided reasons and comments for each item, and these texts were analyzed qualitatively using an affinity diagram, which is also called the Kawakita Jiro (KJ) method [10]. All the qualitative data were segmented into meaningful clauses, then categorized into multiple groups, and after repeated examinations, all groups were converged until theoretical saturation was attained.

This study was conducted in accordance with the principles of the Declaration of Helsinki and was approved by the Hino Hospital ethics committee (No. 2019-3).

\section{Statistical analysis}

All data were expressed as mean and standard deviation, or median and range. For comparison between two groups, continuous variables were analyzed using the Student's $t$-test and categorical variables were analyzed using the chi-square test. Also, the VAS was analyzed using the Wilcoxon rank-sum test. All analysis was performed using the STATA version 15 (STATA Corp. Tx) and Microsoft Excel version 16.26 (Japan Microsoft Corp., Tokyo, Japan).

\section{Results}

Table 1 shows baseline data of working physicians and inpatients under the single-attending-physician system and the multiple-attending-physicians system. There were no significant differences in physicians' age, gender, and post-graduate-year between the two systems. The numbers of inpatients under the single-attending-physician system and the multiple-attending-physicians system were 592 and 263 , respectively. There were no significant differences in patients' age and gender. However, hospitalization days under the single-attending-physician system were significantly longer than those under the multiple-attending-physicians system. Significant differences in living areas and status before admission were also observed between the two systems $(p=0.006$ and $p=0.0014$, respectively). Patients living in Kofu, which is situated just north of Hino, or nursing homes or geriatric facilities were significantly more under the multiple-attending-physicians system than under the single-attendingphysician system. 
Table 1

Baseline data of physicians and inpatients under the single-attending-physician system and the multipleattending-physicians system

\begin{tabular}{|c|c|c|c|c|c|}
\hline \multirow{2}{*}{\multicolumn{2}{|c|}{ number of physicians }} & $\begin{array}{l}\text { Single-attending- } \\
\text { physician system }\end{array}$ & $\begin{array}{l}\text { Multiple-attending- } \\
\text { physicians system }\end{array}$ & \multicolumn{2}{|c|}{$P$ value } \\
\hline & & 5 & 6 & & \\
\hline \multicolumn{2}{|c|}{ age of physicians } & $48(31-60)$ & $40(30-58)$ & \multicolumn{2}{|l|}{0.78} \\
\hline \multicolumn{2}{|l|}{ Male physician } & $4(80.0 \%)$ & $5(83.3 \%)$ & \multicolumn{2}{|l|}{0.89} \\
\hline \multicolumn{2}{|c|}{ Post-graduate-year } & $8(7-28)$ & $15.5(5-35)$ & \multicolumn{2}{|l|}{0.52} \\
\hline \multicolumn{2}{|c|}{ Number of inpatients } & 592 & 263 & \\
\hline \multicolumn{2}{|l|}{ patients' age } & $83.1 \pm 12.2$ & $84.1 \pm 12.3$ & \multicolumn{2}{|l|}{0.86} \\
\hline \multicolumn{2}{|c|}{ Number of male patients } & $260(43.9 \%)$ & $119(45.2)$ & \multicolumn{2}{|l|}{0.71} \\
\hline \multicolumn{2}{|c|}{ Hospitalization days } & $36.9 \pm 60.2$ & $25.1 \pm 29.8$ & \multicolumn{2}{|l|}{0.001} \\
\hline \multirow{5}{*}{$\begin{array}{l}\text { Living areas } \\
\text { of patients }\end{array}$} & Hino Town & $295(49.8 \%)$ & $114(43.3 \%)$ & 0.08 & \multirow[t]{5}{*}{0.006} \\
\hline & Nichinan Town & $44(7.4 \%)$ & $13(4.9 \%)$ & 0.17 & \\
\hline & Kofu Town & $163(27.5 \%)$ & 105(39.9\%) & $<.001$ & \\
\hline & $\begin{array}{l}\text { Outside Hino } \\
\text { County }\end{array}$ & $52(8.7 \%)$ & $15(5.7 \%)$ & 0.12 & \\
\hline & $\begin{array}{l}\text { Outside Tottori } \\
\text { Prefecture }\end{array}$ & $38(6.4 \%)$ & $16(6.0 \%)$ & 0.85 & \\
\hline \multirow{5}{*}{$\begin{array}{l}\text { Status before } \\
\text { admission }\end{array}$} & Outpatient & $413(69.8 \%)$ & $167(63.5 \%)$ & 0.08 & \multirow[t]{5}{*}{0.001} \\
\hline & Referred & $58(9.8 \%)$ & $33(12.6 \%)$ & 0.07 & \\
\hline & Transferred & $45(7.7 \%)$ & $12(4.6 \%)$ & 0.10 & \\
\hline & $\begin{array}{l}\text { Nursing home or } \\
\text { geriatric facility }\end{array}$ & $60(10.2 \%)$ & $48(18.3 \%)$ & 0.001 & \\
\hline & other & $1(0.2 \%)$ & $3(1.2 \%)$ & 0.055 & \\
\hline
\end{tabular}

Table 2 shows the frequency of inpatients' diseases under the two systems. Pneumonia and heart failure were the most frequent under the two systems, and eight out of the top ten diseases were similar under the two systems. Therefore, no differences in the diseases of patients were observed between the two groups. 
Table 2

Ranking and the frequency of inpatients' diseases

Single-attending-physician system

\begin{tabular}{|c|c|c|c|c|c|c|c|}
\hline Rank & Disease & Number & $\begin{array}{l}\text { Proportion } \\
(\%)\end{array}$ & Rank & Disease & Number & $\begin{array}{l}\text { Proportion } \\
(\%)\end{array}$ \\
\hline 1 & Pneumonia & 115 & 19.4 & 1 & Pneumonia & 63 & 24.0 \\
\hline 2 & Heart Failure & 35 & 5.9 & 2 & Heart Failure & 20 & 7.6 \\
\hline 3 & $\begin{array}{l}\text { Urinary Tract } \\
\text { Infection }\end{array}$ & 21 & 3.5 & 3 & $\begin{array}{l}\text { Cerebral } \\
\text { Infarction }\end{array}$ & 9 & 3.4 \\
\hline 4 & Rehabilitation & 14 & 2.4 & 4 & $\begin{array}{l}\text { Failure to } \\
\text { Thrive }\end{array}$ & 9 & 3.4 \\
\hline 5 & Dehydration & 13 & 2.2 & 5 & $\begin{array}{l}\text { Bowel } \\
\text { Obstruction }\end{array}$ & 4 & 1.5 \\
\hline 5 & $\begin{array}{l}\text { Cerebral } \\
\text { Infarction }\end{array}$ & 13 & 2.2 & 5 & Rehabilitation & 4 & 1.5 \\
\hline 7 & $\begin{array}{l}\text { Bowel } \\
\text { Obstruction }\end{array}$ & 11 & 1.9 & 7 & $\begin{array}{l}\text { Impaired } \\
\text { Consciousness }\end{array}$ & 4 & 1.5 \\
\hline 7 & $\begin{array}{l}\text { Colorectal } \\
\text { Polyp }\end{array}$ & 11 & 1.9 & 7 & $\begin{array}{l}\text { Urinary Tract } \\
\text { Infection }\end{array}$ & 8 & 3.0 \\
\hline 9 & Heat Stroke & 9 & 1.5 & 7 & Cellulitis & 4 & 1.5 \\
\hline 10 & $\begin{array}{l}\text { Failure to } \\
\text { Thrive }\end{array}$ & 8 & 1.4 & 7 & $\begin{array}{l}\text { Alcoholic Liver } \\
\text { Disease }\end{array}$ & 3 & 1.1 \\
\hline 11 & $\begin{array}{l}\text { Sleep Apnea } \\
\text { Syndrome }\end{array}$ & 7 & 1.2 & 7 & Appetite loss & 3 & 1.1 \\
\hline 11 & Diabetes & 7 & 1.2 & 7 & Dehydration & 3 & 1.1 \\
\hline 13 & $\begin{array}{l}\text { Acute } \\
\text { Bronchitis }\end{array}$ & 6 & 1.0 & 7 & $\begin{array}{l}\text { Cerebral } \\
\text { Hemorrhage }\end{array}$ & 3 & 1.1 \\
\hline 13 & Dementia & 6 & 1.0 & 7 & Lung Cancer & 3 & 1.1 \\
\hline 13 & Lung Cancer & 6 & 1.0 & 7 & $\begin{array}{l}\text { Pulmonary } \\
\text { Tuberculosis }\end{array}$ & 3 & 1.1 \\
\hline 13 & $\begin{array}{l}\text { Chronic } \\
\text { Kidney } \\
\text { Disease }\end{array}$ & 6 & 1.0 & 16 & $\begin{array}{l}\text { Peripheral } \\
\text { Vertigo }\end{array}$ & 3 & 1.1 \\
\hline
\end{tabular}

Table 3 compares the clinical performance between the single and multiple-attending-physicians system. The average numbers of daily inpatients under the single and multiple-attending-physicians systems were 37 and 11, respectively, while the average numbers of working physicians per day were 3.21 and 1.87, respectively. The average number of patients under the single-attending-physician (11.52 per 
physician and per day) was significantly higher than that under the multiple-attending-physicians system (5.88) $(p<0.01)$. Averaged inpatient care cost per patient under the multiple-attending-physicians group was 712,684 Japanese Yen (6,479 US dollars), lower than that under the single-attending-physician group, which was 1,038,020 Japanese Yen (9,437 US dollars). There was a significant difference in patients' outcome between both systems $(p=0.0071)$. Patients discharged to nursing homes or geriatric facilities were significantly more under the multi-attending-physicians group than under the singleattending-physician group $(p<0.01)$.

Table 3

The comparison of clinical performance between the single and multiple-attending-physicians system

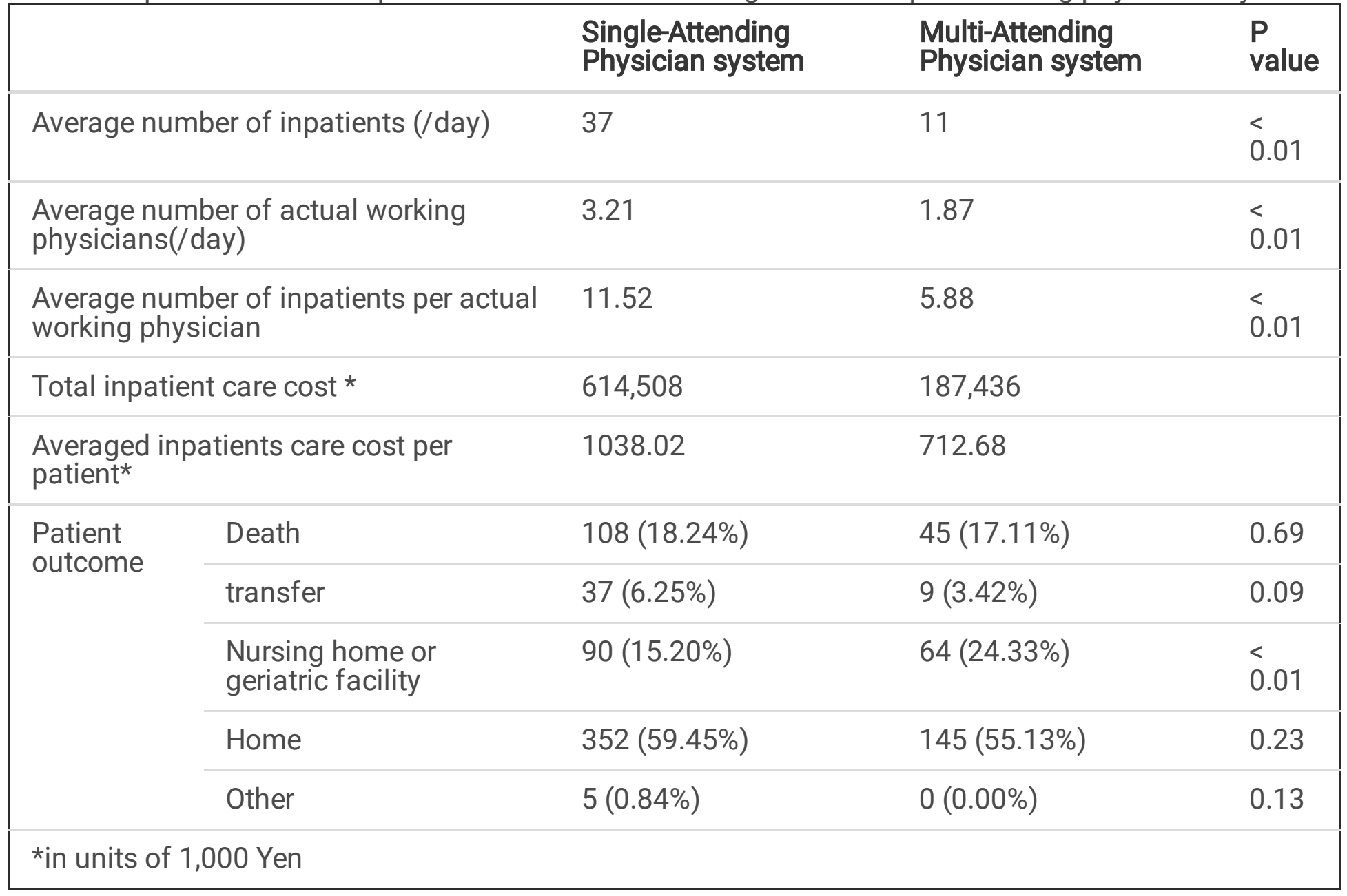

Figure 1 illustrates the degrees of physicians' working conditions assessed by a visual analog scale.

All the 11 physicians engaged under the single or multiple- attending-physicians system answered the questionnaire. The results of the questionnaire survey showed that there was no significant difference between the two groups in all categories. However, physicians under the multiple-attending-physicians system tended to have less off-duty working hours and less physical work burden, while the psychiatric work burden under the two systems seems almost equal.

In the Table 4, we described the advantages and disadvantages of two systems extracted from the data of qualitative survey. 
Table 4

Advantages and disadvantages of ward management under the multiple-attending-physicians system.

Items

Advantages
1) Improvement of physicians' QOL

\section{Descriptions of answers obtained in the survey}

Secured time "I can easily set a schedule and work in a planned manner."

“I didn't want to engage in ward management because it seemed to be impossible in the child-raising years, but the system has relieved my stress and enabled me to engage in it comfortably."

Risk diversification "By sharing responsibilities, I could reduce my mental burden."

2) Lifelonglearning effect

“Other physicians' viewpoints can provide feedback about my practice."

"I could observe my habits in clinical practice."

3) Improving the quality of medical care

Disadvantages

1) Risk of miscommunication
Team communication "Dr. XX conducted examinations of patients instead of me when I forgot to do them."

"Other physicians sometimes identified a clinical condition that I could not find."

Skill compensation "Each physician's strengths are applied to the patient, and the weak points can be covered by other physicians."

Standardization of medical care "As they are viewed by other physicians, the contents of medical records are standardized."

Prevention of medical error "Since other physicians also commit same patients, oversight in medical care will be reduced."

Physician-patient relationship "It is difficult to build a relationship with patients and their family before they are discharged."

Communication among physicians "I missed examinations because I was not notified of their orders."

"I was unsure about what to explain to a patient's family because I was not informed of what they had already been told."

Interprofessional work "There may be confusion among nurses regarding the person they should ask about this patient."

Difficulty in patient understanding "It takes time to grasp the whole condition of a patient whom I met after a few days." 
2) Conflicting treatment policies among physicians
3) Patients' unfamiliarity with multiple-attending physician system

\section{Disagreement of treatment plan}

"A patient whom I thought we could treat at our hospital was soon transferred to a university hospital."

\section{Power relationship among physicians}

"Unbalanced power in the team creates an atmosphere where members hesitate to give their opinion."

"I tend to make requests to physicians who are more approachable, and I often can't help but carry forward problems."

"I tend to make requests to physicians who are more approachable, and I often can't help but carry forward problems."

\section{Lack of leadership}

"Sometimes it's hard to see who is responsible."

"There is a possibility of imposing responsibilities and decision making to a certain person."

"I am anxious about how the patient reacts to the team system because the patient responds the independent medical physician system as a matter of course."

The analysis of the free description of the 11 physicians from single and multiple-attending-physicians systems showed that the multiple-attending-physicians system had three advantages: 1) improvement of physicians' quality of Life (QOL), 2) lifelong-learning effect, and 3) improvement of the quality of medical care. The 3 disadvantages which were identified included: 1) risk of miscommunication, 2) conflicting treatment policies among physicians, and 3 ) unfamiliarity with the culture of the multiple-attendingphysicians system (Table 4).

Furthermore, the category "Improvement of physicians' QOL" was sorted into secured time and risk diversification, and the category "improving the quality of medical care" was sorted into team communication, skill compensation, standardization of medical care, and prevention of medical error. There were conspicuous opinions that the multiple-attending-physicians system provides learning opportunities, especially for physicians raising children and those who are not confident about their skills, provides time support, and contributes to improving the medical care level for patients.

Regarding the disadvantages of the multiple-attending-physicians system, the risk of miscommunication is divided into 4 subcategories: doctor-patient relationship, communication among physicians, interprofessional work, and difficulty in patient understanding. Conflicting treatment policies among physicians is also divided into 3 subcategories: disagreement regarding the treatment plan, power 
relationship among physicians, and lack of leadership. The risk of miscommunication is a problem highlighted not only in the communication between physicians sharing patients' information but also in communication with all related individuals such as patients and nurses. Some physicians complain about stress due to conflict regarding the treatment plan. In case there is a power gradient due to age or social position, the power relationship may interfere with appropriate clinical practice. The third category, unfamiliarity with the culture of the multiple-attending-physicians system, can be considered as a problem peculiar to Japan where the single-attending-physician system is recognized as natural by patients and co-medical staff like nurses. Some physicians worry about patients' feelings for this unfamiliar style of clinical practice.

\section{Discussion}

In this study, we evaluated the clinical performance for inpatients between the single-attending-physician system and the multiple-attending-physicians system cross-sectionally and quantitatively at a rural hospital in Japan. Additionally, we qualitatively analyzed the physicians' work burden under these two attending-physician systems to identify the advantages and disadvantages of the systems.

So far, only a few studies have reported the effects of the multiple-attending-physicians system, and most of them have emphasized the diagnostic accuracy regarding the interpretation of skin lesions or X-ray films [11-15]. In the present study, we obtained data on not only the working hours in the single and multiple-attending-physician systems but also the actual condition of medical care and the burden on physicians in Japan.

In the present study, the length of hospital stay was significantly lower in the multiple-attendingphysicians group than the single-attending-physician group, and inpatient care cost was also lower in the multiple-attending-physicians group. However, we could not calculate significant differences because of the limitation in data collection. The following reasons can be assumed for the decrease in length of hospital stays and the seemingly lower medical costs. First, in the multiple-attending-physicians system, the sharing and discussion of patient information within the attending team may have reduced unnecessary tests and treatments and even facilitated early discharge from the hospital. As a result, unnecessary healthcare costs were reduced and the length of hospital stay was shortened, resulting in a decrease in inpatient care costs.

In this study, there were no significant differences in patients' age, sex, and disease variations between the two attending-physician groups. Although the number of inpatients differed between the two attending-physician groups, the workload per actual working physician was hardly comparable for the following reasons: 1) each physician in both groups had many tasks other than inpatient care, such as participation in outpatient and branch clinics, endoscopy/other procedures and clinical internship (100 students per year), 2) since the multiple-attending-physicians group started their inpatient care in April 2017 , the number of admitted patients was initially small. 
Based on the VAS analysis, there was no significant difference in the indicator of working conditions or burdens between the two groups. However, it is possible that the multiple-attending-physicians group may have fewer working hours and less physical workload but may experience some difficulty regarding understanding the patients' disease status, doctor-patient relationship, imbalances due to workload, and inter-professional relationships than the single-attending-physician group. Notably, the single-attendingphysician system tended to be more physically stressful for the physicians. On the other hand, both the groups had similar scores on psychiatric burden. The multiple-attending-physician system may have less burden of overtime work than the single-attending-physician system, but there are more difficulties in understanding patients and cooperation among other professionals, which may have been offset and resulted in equal psychiatric burden.

The survey showed that the multiple-attending-physicians system has the advantages of 1) improving the physicians' QOL, 2) lifelong learning effect, and 3) improving the quality of medical care. Its disadvantages include 1) the risk of miscommunication, 2) conflicting treatment policies among physicians, and 3) patients' unfamiliarity with the multiple-attending-physician system. While improving the physicians' QOL is the most important benefit, the effects on enhancing life-long learning, which is generally related to physicians' burnout [16], suggests that the multiple-attending-physicians system may be a means to prevent physicians from dropping out in the long term. Furthermore, from the decisionmaking perspective, the involvement of multiple physicians can minimize sanctioning and reduce emotional distress among physicians [17].

On the other hand, there were ambivalent aspects regarding communication and the prevention of mistakes. Multiple rotations of physicians will inevitably increase the number of handovers between physicians, which could increase the risk of miscommunication, and the reduced duration of hospital stay per physician may cause communication troubles with patients and co-medical staff. At the same time, it is indicated that careful observation of patient management by multiple physicians can prevent mistakes and improve the level of overall medical care.

A cross-sectional study demonstrated that with more physicians, the accuracy in clinical practice under the team approach will improve [11]. However, several studies indicate that the involvement of multiple professionals in a patient's care could cause confusion and frustration among physicians, patients, and co-working staff in inpatient care, especially in ICU and end-of-life care [18-22]. Management of the endof-life decision-making varied in relation to multiple and shifting attending responsibilities, and some patient's families and clinicians were confused about who was making patient care decisions and with whom they should confer [18]. Many patients were unable to name anyone when asked to identify an inpatient physician in charge of their care [21], and nearly $50 \%$ of responding attending physicians knew dying patients for less than 48 hours [22]. According to the present study, this might be just a matter of traditional culture or depend on the severity and urgency of the situation, such as the ICU and acute care hospital. To tackle this problem, the standardization of the handoff process is important since only a few physicians engage in the appropriate handoff process [23]. 
The limitations of this study are, first, that the data were obtained from a single center with a small sample. Second, although the two attending-physician groups in this study worked in common practice areas, we did not collect data from patients and co-working staff. Regarding the pros and cons of the multiple-attending-physicians system, feelings and thoughts of patients as beneficiaries of care and coworking staff as counterparts of physicians' work are also important. Third, in addition to the number discrepancy of inpatients between two groups, physicians from each group had different department background, which might affect the interpretation of the results to some degree. Despite the several research limitations, this is one of the few studies that identified the advantages and disadvantages of the two attending-physician systems. Further studies should include the two attending-physician systems in the same department or use a larger sample size in a multicenter setting and investigate the satisfaction of patients and healthcare professionals other than physicians in the case of the multipleattending-physicians-system. Similarly, research should be conducted on the burden of physicians and the quality of care in outpatient settings in Japan.

\section{Conclusion}

We have acknowledged that the multiple-attending-physicians system may reduce the physical burden of physicians without compromising their clinical performance. This system can improve physicians' QOL, encourage lifelong learning, and improve the quality of medical care, and may contribute to maintaining medical services at local medical facilities, which have difficulties in securing full-time physicians.

\section{Abbreviations}

QOL: quality of life VAS: visual analog scale KJ: Kawakita Jiro

\section{Declarations}

\section{Acknowledgements}

We would like to express our appreciation to the entire staff of the Hino Hospital who were willing to provide data and opportunity to assess clinical practice.

\section{Ethics declarations}

\section{Ethics approval}

This study was conducted in accordance with the principles of the Declaration of Helsinki and was approved by the Hino Hospital ethics committee (No. 2019-3). At the start of this study, written informed consent was obtained from all physicians who were surveyed, and allowed that they could withdraw their consent or ask questions at any time.

\section{Consent for publication}


Not applicable.

\section{Competing interests}

All authors declare no competing interests.

\section{Availability of data and materials}

The datasets used during the current study are available from the corresponding author on reasonable request.

\section{Author Contributions}

Project administration: Shin-ichi Taniguchi, Masahiko Koda

Conceptualization: Daeho Park, Shin-ichi Taniguchi

Methodology: Daeho Park, Shin-ichi Taniguchi, Masahiko Koda

Data curation: Toshihiro Hamada, Masahiko Koda, Daeho Park

Writing - original draft: Daeho Park, Toshihiro Hamada

Writing - review \& editing: all authors

\section{References}

1. Matsumoto M, Kimura K, Inoue K, Kashima S, Koike S, Tazuma S. Aging of hospital physicians in rural Japan: A longitudinal study based on national census data. PLoS One. 2018 Jun 1;13(6):e0198317.

2. O'Dea B, O'Connor P, Lydon S, Murphy AW. Prevalence of burnout among Irish general practitioners: a cross-sectional study. Ir J Med Sci. 2017 May;186(2):447-453.

3. Galam E, Komly V, Le Tourneur A, Jund J. Burnout among French GPs in training: a cross-sectional study. Br J Gen Pract. 2013 Mar;63(608):e217-24.

4. Riley R, Spiers J, Buszewicz M, Taylor AK, Thornton G, Chew-Graham CA. What are the sources of stress and distress for general practitioners working in England? A qualitative study. BMJ Open. 2018 Jan 11;8(1):e017361.

5. The Panel on Reform of Doctors' Work Style under the Ministry of Health, Labour and Welfare. 2018. [Available from : https://www.mhlw.go.jp/stf/shingi/other-isei_469190.html] (accessed 2018-12-01)

6. The 188th General Meeting of the Central Social Insurance Medical Council. Reduction of burdens of healthcare professionals at hospitals (No.1) (March 2, 2011).

7. Takemura T, Shimada M, Kataoka Y, Iki R, Hirai T. Prospective, transverse study on the effects of multiple-attending-physicians system on work hours, job satisfaction and burnout of hospital 
doctors. Hospital [0385-2377]. 2018;77(10):806 -811

8. Dreher A, Theune M, Kersting C, Geiser F, Weltermann B. Prevalence of burnout among German general practitioners: Comparison of physicians working in solo and group practices. PLoS One. 2019 Feb 6;14(2):e0211223.

9. Wewers ME, Lowe NK. A critical review of visual analogue scales in the measurement of clinical phenomena. Res Nurs Health. 1990 Aug;13(4):227-36.

10. Kawakita J. The way of thinking for creative development. Tokyo: Chuukousinnsyo, 1967.

11. Barnett ML, Boddupalli D, Nundy S, Bates DW. Comparative Accuracy of Diagnosis by Collective Intelligence of Multiple Physicians vs Individual Physicians. JAMA Netw Open. 2019 Mar 1;2(3):e190096.

12. Wolf M, Krause J, Carney PA, Bogart A, Kurvers RH. Collective intelligence meets medical decisionmaking: the collective outperforms the best radiologist. PLoS One. 2015 Aug 12;10(8):e0134269.

13. Kurvers RH, Krause J, Argenziano G, Zalaudek I, Wolf M. Detection Accuracy of Collective Intelligence Assessments for Skin Cancer Diagnosis. JAMA Dermatol. 2015 Dec 1;151(12):1346-1353.

14. Kurvers RH, Herzog SM, Hertwig R, Krause J, Carney PA, Bogart A, ArgenzianoG,Zalaudek I, Wolf M. Boosting medical diagnostics by pooling independent judgments. Proc Natl AcadSci U S A. 2016 Aug 2;113(31):8777-82.

15. Kämmer JE, Hautz WE, Herzog SM, Kunina-Habenicht O, Kurvers RHJM. The Potential of Collective Intelligence in Emergency Medicine: Pooling Medical Students' Independent Decisions Improves Diagnostic Performance. Med Decis Making. 2017 Aug;37(6):715-724.

16. West CP, Dyrbye LN, Shanafelt TD. Physician burnout: contributors, consequences and solutions. J Intern Med. 2018 Jun;283(6):516-529.

17. El Zein M, Bahrami B, Hertwig R. Shared responsibility in collective decisions. Nat Hum Behav. 2019 Jun;3(6):554-559.

18. Baggs JG, Schmitt MH, Prendergast TJ, Norton SA, Sellers CR, Quinn JR, Press N. Who is attending? End-of-life decision making in the intensive care unit. J Palliat Med. 2012 Jan;15(1):56-62.

19. Chaitin E, Stiller R, Jacobs S, Hershl J, Grogen T, Weinberg J. Physician-patient relationship in the intensive care unit: erosion of the sacred trust? Crit Care Med. 2003 May;31(5 Suppl):S367-72.

20. Morgenstern L. A piece of my mind. Will the real doctor please stand up? JAMA.2003 Jan 1;289(1):18.

21. Arora V, Gangireddy S, Mehrotra A, Ginde R, Tormey M, Meltzer D. Ability of hospitalized patients to identify their in-hospital physicians. Arch Intern Med. 2009 Jan 26;169(2):199-201.

22. Galanos AN, Morris DA, Pieper CF, Poppe-Ries AM, Steinhauser KE. End-of-life care at an academic medical center: are attending physicians, house staff, nurses, and bereaved family members equally satisfied? Implications for palliative care. Am J HospPalliat Care. 2012 Feb;29(1):47-52.

23. Lane-Fall MB, Collard ML, Turnbull AE, Halpern SD, Shea JA. ICU Attending Handoff Practices: Results From a National Survey of Academic Intensivists. Crit Care Med. 2016 Apr;44(4):690-8. 


\section{Figures}

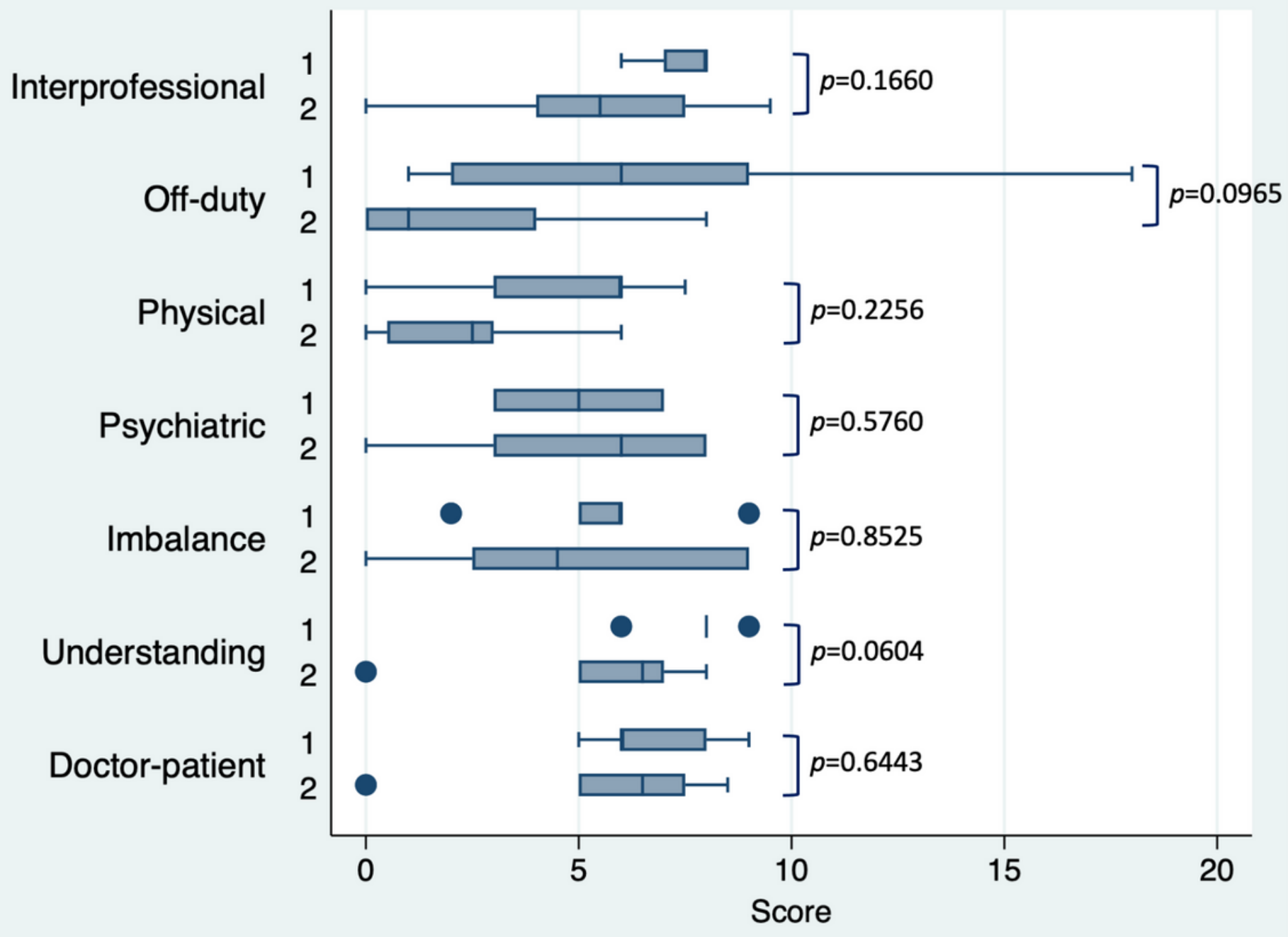

\section{Figure 1}

Results of the questionnaire using the VAS. Shown are the interview results of both the attending physician groups. 1 and 2 denotes the single and multiple-attending-physicians group, respectively. Questions related to current working situations assessed by a visual analog scale (VAS) scored from 0 to 10 are followings; How well do you collaborate with other professionals?; Interprofessional How much is the physical and psychiatric burden of providing inpatient care?; physical and psychiatric How well do you understand your patients' disease status?; Understanding How much do you have a trusting relationship with your patients?; Doctor-patient How much do you feel an unbalance in your work, such as focusing on yourself or other doctors?; Unbalances Off-duty working hours per week (Off-duty) is asked by following question; How many hours per week are spent on after-hours inpatient services? (Out-ofhours will be considered before 8:30 a.m. and after 5 p.m.); Off-duty These boxes show interquartile range with vertical line inside meaning medium. The horizontal lines extending from the box represent the 
maximum and minimum values excluding the outlier, and the dots represents the outliers which are more than 1.5 times the upper and lower quartiles. 